\title{
Nucleosynthesis in AGB Stars: the Role of Dredge-Up and Hot Bottom Burning
}

\author{
John Lattanzio \\ Centre for Stellar and Planetary Astrophysics, Monash University, \\ 3800, Australia, and \\ Institute for Nuclear Theory, University of Washington, Seattle, USA
}

\begin{abstract}
We review the basics of AGB nucleosynthesis in thermal pulses, in the hydrogen burning shell, and by Hot Bottom Burning.
\end{abstract}

\section{Introduction}

Planetary nebulae (PNe) are perhaps the most visible legacy of the last stages of the life of most stars, certainly those with low and intermediate masses (initially about $1-8 \mathrm{M}_{\odot}$, depending on the composition). They thus reflect the composition of their immediate progenitors, the Asymptotic Giant Branch (AGB) stars. Hence a thorough understanding of AGB stars is needed as a context for questions about PNe compositions. Planetary nebulae offer us an opportunity to probe the composition of the inner layers of AGB stars, either through the last thermal pulses or via the central star, which has now lost its envelope and reveals the products of helium burning. A quantitative understanding is still beyond us, but we have a good qualitative understanding, which will be summarised here.

Evolution to the start of the AGB is fairly well understood, although there are still problems. Aside from quantitative problems, there are even fundamental problems such as the presence and extent of convective overshoot and other mixing. This is all within the context of "standard" or "canonical" models which do not include the effects of rotation, or other physics usually neglected (eg magnetic fields). It is a lucky fact that non-rotating one-dimensional models fit most observations very well, telling us that rotation is largely unimportant.

1 For our purposes it is sufficient to start with a star at the end of core helium burning, about to begin its AGB phase of evolution.

\section{AGB Evolution}

\subsection{The Early-AGB}

Figure 1 shows the structure at the start of the AGB phase. The envelope still has mostly the original composition of the star. The first dredge-up event on the giant branch will have increased the surface content of ${ }^{13} \mathrm{C}$ and ${ }^{14} \mathrm{~N}$, and

\footnotetext{
${ }^{1}$ except for the cases where it is important...
} 


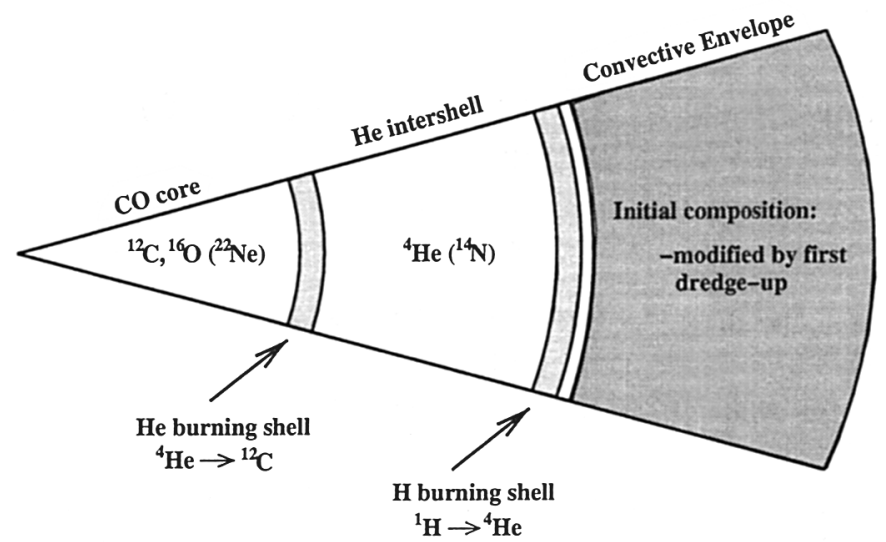

Figure 1. Structure and main composition at the start of the AGB phase. The most abundant trace element is in parentheses.

it is likely that rotationally driven mixing will have increased the effect of this beyond what is predicted in non-rotating models (Charbonnel 1995). Moving inward we come to the hydrogen burning shell (HBS) which is burning $\mathrm{H}$ into $\mathrm{He}$ via the CNO cycles. Consequently below the HBS the composition is primarily ${ }^{4} \mathrm{He}$ and ${ }^{14} \mathrm{~N}$ since the CNO cycles convert essentially all CNO nuclei into ${ }^{14} \mathrm{~N}$. Below the intershell region is the helium burning shell (HeBS) which burns $\mathrm{He}$ into ${ }^{12} \mathrm{C}$. However, core $\mathrm{He}$ burning has converted the He into ${ }^{12} \mathrm{C}$ and ${ }^{16} \mathrm{O}$ in proportions which depend on the exact rate of the ${ }^{12} \mathrm{C}(\alpha, \gamma){ }^{16} \mathrm{O}$ reaction, but for currently believed rates they are about equal in abundance. Note that core burning produces more ${ }^{16} \mathrm{O}$ than shell burning because there is convection to bring fresh ${ }^{4} \mathrm{He}$ into contact with the ${ }^{12} \mathrm{C}$ produced, which does not happen during shell burning. Note also that two $\alpha$-captures on the relatively abundant ${ }^{14} \mathrm{~N}$ has produced ${ }^{22} \mathrm{Ne}$ : one ${ }^{22} \mathrm{Ne}$ nucleus for each of the initial CNO nuclei present.

As the star ascends the AGB the He shell is ignited, and burns its way through the profile left over from core helium burning. If the star is sufficiently massive (exceeding about $4 \mathrm{M}_{\odot}$, depending on composition) then the energy released is so great that the star expands, the HBS is temporarily extinguished, and the convective envelope once again extends into the $\mathrm{He}$ intershell. This is called the second dredge-up event, and it enriches the surface layers in ${ }^{4} \mathrm{He}$ and ${ }^{14} \mathrm{~N}$ primarily. The HBS is again ignited and the star continues its AGB evolution and soon begins thermally pulsing.

\subsection{The Thermally Pulsing AGB}

The combination of a geometrically thin shell and strong temperature dependence ensures that the helium shell is unstable, and it will "flash" periodically (Schwarzschild and Härm 1965, Despain 1981). Each pulse of the HeBS drives a convective region which extends almost to the HBS, as shown in Figure 2a for the "on" phase. This energy input causes an expansion ("power-down" phase) and the HBS is extinguished, allowing convection to extend inwards in mass (the 


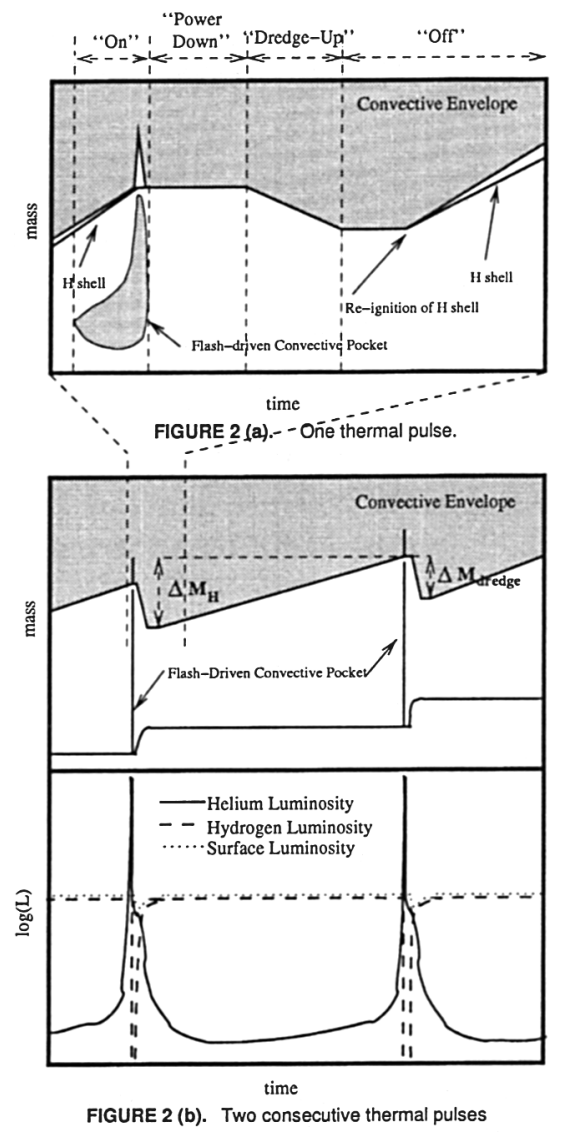

Figure 2. Schematic showing the details of one thermal pulse (upper panel) and two consecutive pulses (lower panels).

"dredge-up" phase). The HBS re-ignites and the evolution continues (the "off" phase). Note that most of the following interpulse time is spent with the HBS providing the energy (Figure 2b): it is only during the pulses that the HeBS burns substantially.

In this way the shells alternately progress outward through the star, and such evolution continues until the $\mathrm{CO}$ core exceeds the Chandrasekhar limit or the HBS reaches the surface. Usually it is the latter, or rather, the effect of mass-loss is to ensure that the surface moves inward (in mass) to meet the HBS! Thus it is mass-loss which is usually responsible for terminating the AGB evolution, and there is good evidence now for a very rapid "super-wind" phase at the end of the AGB (eg Schröder, Winters and Sedlmayr 1999). It is here that the AGB ends and the PN phase begins, but let us now return to the AGB and investigate the nucleosynthesis which occurs there. 


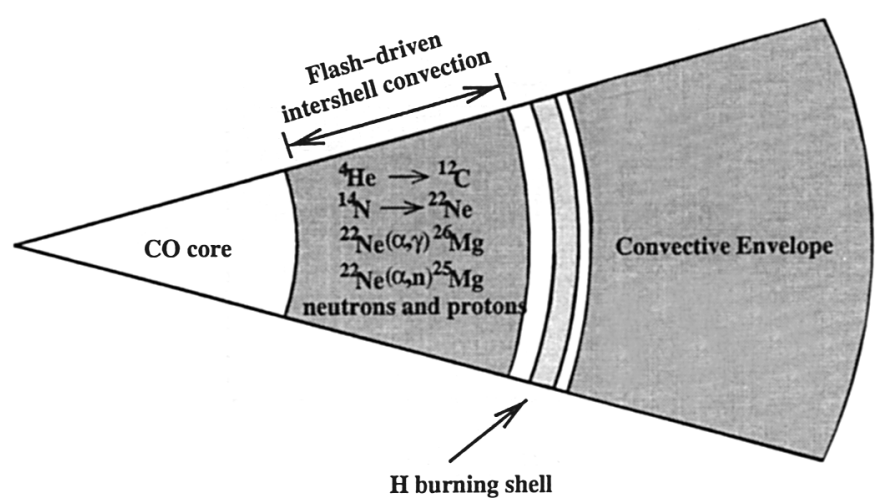

Figure 3. Main nucleosynthesis during a thermal pulse.

\section{Nucleosynthesis in Thermal Pulses}

\subsection{The Pulse itself}

The main cauldron in AGB stars is the HeBS during a shell flash, with temperatures reaching several hundred million degrees for the more massive AGB stars, and at least 100 million for the lower masses. Apart from ${ }^{4} \mathrm{He}$ burning into ${ }^{12} \mathrm{C}$ much else occurs in this convective shell, as illustrated in Figure 3 . The ${ }^{14} \mathrm{~N}$ left over from CNO cycling in the HBS is converted to ${ }^{22} \mathrm{Ne}$ via $\alpha$-captures. If the temperature is high enough (ie in the more massive AGB stars, above about $3 \mathrm{M}_{\odot}$ ) then the ${ }^{22} \mathrm{Ne}$ is also subject to $\alpha$-captures producing either ${ }^{25} \mathrm{Mg}$ or ${ }^{26} \mathrm{Mg}$ depending on whether the reaction is and $(\alpha, \mathrm{n})$ or $(\alpha, \gamma)$ respectively. So we expect PNe to be enriched in ${ }^{22} \mathrm{Ne}$ (at the level of the initial CNO nuclei) if they are not massive enough to initiate $\alpha$-captures on the ${ }^{22} \mathrm{Ne}$.

Note that if ${ }^{22} \mathrm{Ne}$ does capture $\alpha$ particles then there are neutrons released, which can take part not only in $(n, \gamma)$ reactions but also $(n, p)$ as well, thus releasing protons.

\subsection{The Role of the Hydrogen Shell}

Figure 2 shows that the envelope material is processed by the HBS during the interpulse phase. As the envelope contains material dredged-up from the intershell, this means that the products of helium burning in the intershell are then exposed to proton captures in the HBS. This material is then engulfed by the intershell convection at the next pulse (where further processing may occur!) and is then dredged to the surface where the changes in composition become visible. The main species produced in this way are ${ }^{23} \mathrm{Na},{ }^{26} \mathrm{Al}$ and small amounts of ${ }^{27} \mathrm{Al}$

We have seen that the intershell region is rich in ${ }^{22} \mathrm{Ne}$ and, for more massive AGB stars, also the heavy $\mathrm{Mg}$ isotopes. Once this material is mixed to the surface it is burned by the HBS, and the Ne-Na cycle can make (primary!) ${ }^{23} \mathrm{Na}$ from ${ }^{22} \mathrm{Ne}(\mathrm{p}, \gamma){ }^{23} \mathrm{Na}$ (see Mowlavi 1999b). Enrichments up to $0.5 \mathrm{dex}$ are expected, and should be higher at lower metallicities, of course. 
Similarly, the ${ }^{25} \mathrm{Mg}$ and ${ }^{26} \mathrm{Mg}$ produced in the intershell region are dredged to the surface and processed by the HBS. Here the $\mathrm{Mg}-\mathrm{Al}$ cycle adds protons to these to produce ${ }^{26} \mathrm{Al}$ and ${ }^{27} \mathrm{Al}$, respectively. The ${ }^{26} \mathrm{Al}$ eventually decays to ${ }^{26} \mathrm{Mg}$ over a few million years, which exceeds the AGB lifetime. However the biggest hurdle to the production of larger amounts of ${ }^{26} \mathrm{Al}$ is that it is a very efficient neutron poison, so that when engulfed by the intershell convective region at the next pulse, it experiences ${ }^{26} \mathrm{Al}(\mathrm{n}, \mathrm{p})^{26} \mathrm{Mg}$. The details are rather complicated, depending on the shell temperature, and the reader is referred to Mowlavi and Meynet (2000) for details.

\subsection{Dredge-up and the Carbon Pocket}

During the power-down phase the star expands and cools, allowing for dredge-up of the newly synthesized material. However strange things happen, making the details elusive. For example, a strict application of the Schwarzschild criterion can find a "boundary" for the convective region, but it is an unstable boundary (Frost and Lattanzio 1996). Nevertheless, if it is used as the boundary, then often no dredge-up will result (Mowlavi 1999a). The amount of dredge-up found in models depends on how one handles the interaction between iterations for the structure of the star and mixing within the convective regions: both affect the position of the convective boundary and hence the depth of dredge-up. The process is complex, often showing behaviour similar to semiconvection, as first noticed by Iben and Renzini (1982).

One of the consequences of such partial mixing is that some protons from the convective envelope are deposited in small amounts in the carbon-rich intershell region. Then when the star heats again the protons are captured by the ${ }^{12} \mathrm{C}$ to form ${ }^{13} \mathrm{C}$. If there are too many protons then the $\mathrm{CN}$ cycle continues with the ${ }^{13} \mathrm{C}$ burning further to ${ }^{14} \mathrm{~N}$. But as long as there is a region of very low proton concentration, then a pocket enriched in ${ }^{13} \mathrm{C}$ will form (usually next to a similar ${ }^{14} \mathrm{~N}$ pocket where the protons were more abundant). This is illustrated in Figure 4. Note that any diffusion process can create such a partially mixed region, and rotation is another possibility (see Herwig elsewhere in this volume).

From the point of view of nucleosynthesis, the appearance of some ${ }^{13} \mathrm{C}$ is crucial, because further heating during the interpulse phase will ignite the reaction ${ }^{13} \mathrm{C}(\alpha, n){ }^{16} \mathrm{O}$ which releases neutrons where the ${ }^{13} \mathrm{C}$ pocket used to be. These neutrons are substantially captured by various iron-peak elements present in the star, and thus produce "s-process" elements. At the next pulse, this shell of s-process elements will be engulfed by the intershell convection, and then dredged to the surface at the next dredge-up phase. The details of the s-process do not concern us here, and the reader is referred to the papers by the Torino group (eg Busso et al 2001, Busso, Gallino and Wasserburg 1999).

\subsection{Fluorine Production}

Thermally pulsing AGB stars have also been unambiguously identified as a source of ${ }^{19} \mathrm{~F}$ (Jorissen, Smith and Lambert 1992). The starting point is once again a neutron source, probably $\alpha$-capture on ${ }^{13} \mathrm{C}$, which occurs during the interpulse phase in the region immediately below the convective envelope (see Figure 4). The abundant ${ }^{14} \mathrm{~N}$ in this region can compete with heavier elements for the neutron, and release protons via ${ }^{14} \mathrm{~N}(\mathrm{n}, \mathrm{p}){ }^{14} \mathrm{C}$. We have also seen that there 


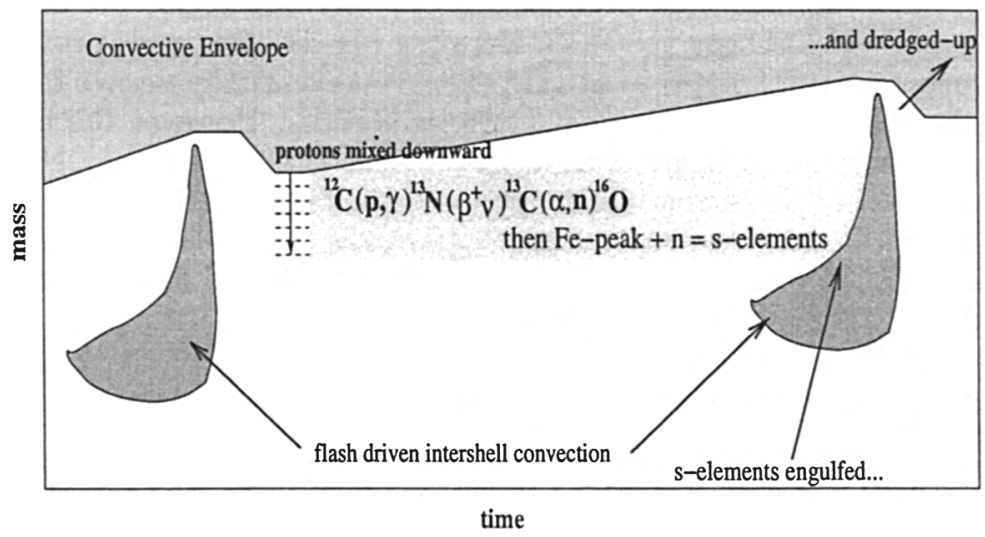

Figure 4. Formation of the Carbon-pocket and subsequent neutron captures

may be some ${ }^{26} \mathrm{Al}$ present, produced by the $\mathrm{Mg}-\mathrm{Al}$ cycle in the HBS, and this is another efficient neutron poison again releasing protons via ${ }^{26} \mathrm{Al}(\mathrm{n}, \mathrm{p}){ }^{26} \mathrm{Mg}$. The important point is that the neutron source has now produced some protons.

Simultaneously, an $\alpha$-capture on ${ }^{14} \mathrm{~N}$ (followed by a $\beta$-decay) produces ${ }^{18} \mathrm{O}$, which can catch some of the protons now present and this gives ${ }^{15} \mathrm{~N}$ via $(\mathrm{p}, \alpha)$ reactions. Finally, ${ }^{15} \mathrm{~N}(\alpha, \gamma){ }^{19} \mathrm{~F}$ produces the fluorine.

This production mechanism is quite complicated, and the site of various stages depends on the temperature (Mowlavi, Jorissen and Arnould 1996). Less massive stars will make the ${ }^{15} \mathrm{~N}$ in the intershell convective region during a pulse, while more massive stars, being hotter, can produce it in the radiative region where the ${ }^{13} \mathrm{C}$-pocket formed. Also, the production can move from the HBS to the intershell as the temperature of these regions changes during evolution along the AGB. Further, as we will see below, ${ }^{19} \mathrm{~F}$ can also be destroyed by hot bottom burning!

\section{Nucleosynthesis by Hot Bottom Burning}

For intermediate mass stars (above about $4 \mathrm{M}_{\odot}$, depending on the composition) the bottom of the convective envelope can extend into the top layers of the HBS, and hence expose the envelope to high temperatures. This is known as Hot Bottom Burning (HBB). The consequences can be dramatic; all the material previously processed and mixed to the surface can now be further processed at quite high temperatures (up to 100 million $\mathrm{K}$ ) during the interpulse phase.

\subsection{Li production}

Earlier hydrogen burning evolution has created some ${ }^{3} \mathrm{He}$ within the star, and when this experiences high temperatures it creates ${ }^{7} \mathrm{Be}$ by the start of the PPII and PPIII chains. If this ${ }^{7} \mathrm{Be}$ can then be mixed to cooler regions it captures an electron to form ${ }^{7} \mathrm{Li}$ : this process is known as the Cameron-Fowler Beryllium Transport Mechanism (Cameron and Fowler 1971). Abundances as high as 
$\log \epsilon\left({ }^{7} \mathrm{Li}\right) \simeq 4.5$ can be formed in this way (Sackmann and Boothroyd 1992) and match the observed super lithium-rich AGB stars in the Magellanic Clouds (Smith and Lambert 1989, 1990). The existence of enhanced ${ }^{7} \mathrm{Li}$ in less luminous giants may indicate some mixing through radiative regions, which is qualitatively similar to the above mechanism (Sackmann and Boothroyd 1999)

Quantitative calculations show that ${ }^{7} \mathrm{Li}$ production begins once the envelope temperature exceeds 50 million $\mathrm{K}$. The duration of the super lithium-rich phase is temporary, because subsequent passes through the bottom of the convective envelope succeed in destroying the ${ }^{7} \mathrm{Li}$, which cannot be replenished once the ${ }^{3} \mathrm{He}$ is all consumed. Whether these stars can alter the galactic ${ }^{7} \mathrm{Li}$ content depends crucially on the timing and extent of the mass-loss (Travaglio et al 2001).

\subsection{CNO cycling and Carbon stars}

If $\mathrm{HBB}$ is present then it can process the $\mathrm{CNO}$ in the envelope. This has many effects: 1) it burns ${ }^{12} \mathrm{C}$ into ${ }^{13} \mathrm{C}$ and ${ }^{14} \mathrm{~N}$; 2) this can stop the star from becoming a Carbon star, by keeping the number ratio $\mathrm{C} / \mathrm{O}$ below unity; 3 ) it can dramatically alter the ${ }^{12} \mathrm{C} /{ }^{13} \mathrm{C}$ ratio, producing values as high as the $\mathrm{CN}$ equilibrium value of about $3.5 ; 4$ ) it can produce large amount of (primary) ${ }^{14} \mathrm{~N}$, especially at low metallicities. Details can be found in Boothroyd, Sackmann and Ahern (1993), and this seems to explain the absence of high luminosity Carbon stars, as these stars are the very ones which experience HBB.

When the envelope mass decreases below some critical amount the HBB will cease. Likewise, dredge-up seems to require a minimum envelope mass. If $\mathrm{HBB}$ stops before dredge-up, as found by Frost et al (1998), then these high luminosity stars may again become carbon rich, but only at the end of their lives when they are no longer optically visible. Such models explain the obscured Carbon stars observed in the Magellanic Clouds by van Loon et al (1998).

The effect of HBB on the oxygen isotopes is discussed in Boothroyd, Sackmann and Wasserburg (1994) while primary nitrogen production is discussed in Lattanzio, Forestini and Charbonnel (2000).

\subsection{The $\mathrm{Ne}-\mathrm{Na}$ and $\mathrm{Mg}-\mathrm{Al}$ cycles}

We have seen how the HBS can process various species by the $\mathrm{Ne}-\mathrm{Na}$ and $\mathrm{Mg}$ Al cycles, producing ${ }^{23} \mathrm{Na}$ from ${ }^{22} \mathrm{Ne}$ and ${ }^{26} \mathrm{Al}$ from ${ }^{25} \mathrm{Mg}$. The same can occur during $\mathrm{HBB}$, and in some cases the effect is much stronger here because material is cycled again and again, through the bottom of the envelope. Hence although small amounts of ${ }^{26} \mathrm{Al}$ and ${ }^{23} \mathrm{Na}$ can be made by the HBS in low mass stars, the occurrence of $\mathrm{HBB}$ in more massive stars can produce much larger amounts of these species. Such seems to be required by some of the high ${ }^{26} \mathrm{Al} /{ }^{27} \mathrm{Al}$ ratios seen in pre-solar meteorite grains (Ámari 1999).

\subsection{Fluorine Destruction}

We have seen how ${ }^{19} \mathrm{~F}$ can be produced in low and intermediate mass AGB stars. But for stars where $\mathrm{HBB}$ is active another problem arises (Mowlavi et al 1996). Once the temperature at the base of the convective envelope exceeds about 70 million $\mathrm{K}$ then proton captures on ${ }^{19} \mathrm{~F}$ can actually destroy the fluorine again: ${ }^{19} \mathrm{~F}(\mathrm{p}, \alpha){ }^{16} \mathrm{O}$ occurs. Thus the full fluorine story is rather complicated, 


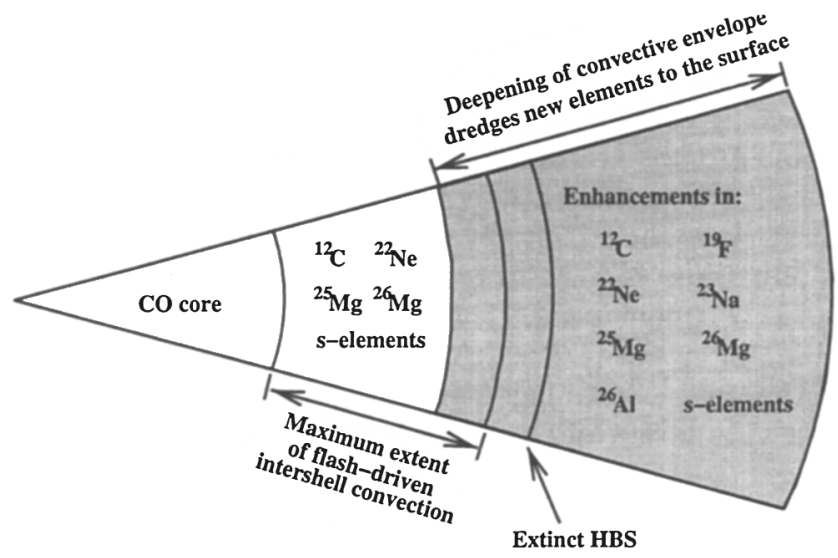

Figure 5. Dredge-up of newly processed material.

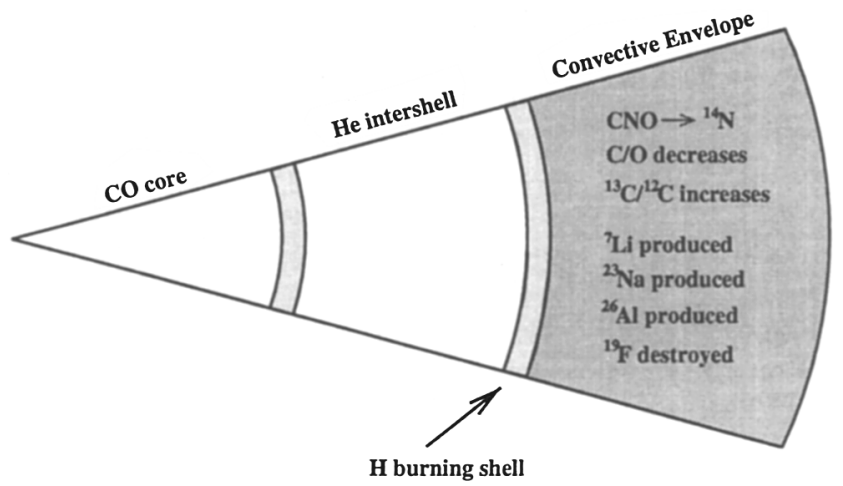

Figure 6. Main effects of Hot Bottom Burning.

with production and destruction occurring at different sites, often within the same star as it evolves along the AGB and the temperatures change.

\section{Summary}

We summarise the main nucleosynthesis from thermal pulsing in Figure 5. This shows the species enriched by thermal pulses and the ensuing dredge-up. Note that each dredge-up episode captures the products of the HBS as well as the Heburning intershell convective region, and brings them to the surface. Similarly, figure 6 summarises the main species produced or destroyed by hot bottom burning. It also shows the most important changes in various isotopic and elemental ratios. 
It is now clear that AGB stars are substantial contributors to galactic chemical evolution. What is really needed is a complete test of the model predictions against observations, which has so far been rather limited and mostly consists of trying to reproduce the Carbon star luminosity function in the Magellanic Clouds. Further, quantitative predictions of abundances depend on dredge-up, which remains a stumbling block for theory.

Acknowledgments. The author would like to thank and acknowledge the hospitality of the Institute for Nuclear Theory and the Department of Astronomy at the Univeristy of Washington, where much of this work was written. He also thanks the editors for their patience and the conference organisers for their efforts.

\section{References}

Amari, S. 1999, In: Bernatowicz J., Zinner E. (eds.) Astrophysical Implications of the Laboratory Study of Presolar Materials. AIP Conf. Proc. 402, 13

Boothroyd, A. I., Sackmann, I.-J. and Ahern, S. C., 1993, ApJ, 416, 762

Boothroyd, A. I., Sackmann, I.-J. and Wasserburg, G. J., 1994, ApJ, 430, L77

Busso, M., Gallino R., and Wasserburg, G. J., 1999, ARA\&A, 37, 239

Busso, M., Gallino R., Lambert, D. L., Travaglio, C., and Smith, V. V., 2001, ApJ, 557, 803

Cameron, A. G. and Fowler, W. A. 1971, ApJ, 164, 111

Charbonnel, C. 1995, ApJ, 453, L41

Frost, C. A. and Lattanzio, J. C., 1996, ApJ, 473, 383

Despain, K. H. 1981, ApJ, 251, 639

Iben, I. Jr., and Renzini, A. 1982, ApJ, 259, L79

Jorissen, A., Smith, V. V. and Lambert, D. L. 1992, A\&A, 261, 164

Lattanzio, J. C., Forestini, M. and Charbonnel C. 2000, Mem Astron Soc Italia, 71, 797

van Loon, J. Th. et al, 1998, A\&A, 329, 169

Mowlavi, N., 1999a, A\&A, 344, 617

Mowlavi, N., 1999b, A\&A, 350, 73

Mowlavi, N., Jorissen, A. and Arnould, M. 1996, A\&A, 311,803

Mowlavi, N., and Meynet, G. 2000, A\&A, 361, 959

Sackmann, I.-J., and Boothroyd, A. I. 1992, ApJ, 392, L71

Sackmann, I.-J., and Boothroyd, A. I. 1999, ApJ, 510, 232

Schröder, K. P., Winters, J. M., and Sedlmayr, E. 1999, A\&A, 349, 898

Schwarzschild, M. and Härm, R. 1965, ApJ, 142, 855

Smith, V. V. and Lambert, D. L., 1989, ApJ, 345, L57

Smith, V. V. and Lambert, D. L., 1990, ApJ, 361, L69

Travaglio, C. et al, 2001, ApJ, 559, 909 